\title{
Acute ischemic stroke in childhood: a comprehensive review
}

\author{
Mario Mastrangelo ${ }^{1} \cdot$ Laura Giordo $^{1} \cdot$ Giacomina Ricciardi $^{1} \cdot$ Manuela De Michele $^{2} \cdot$ Danilo Toni $^{2} \cdot$ Vincenzo Leuzzi $^{1}$
}

Received: 19 February 2021 / Revised: 10 May 2021 / Accepted: 15 July 2021 / Published online: 29 July 2021

(c) The Author(s) 2021

\begin{abstract}
This review provides an updated analysis of the main aspects involving the diagnosis and the management of children with acute ischemic stroke. Acute ischemic stroke is an emergency of rare occurrence in children (rate of incidence of 1/3500 live birth in newborns and 1-2/100,000 per year during childhood with peaks of incidence during the perinatal period, under the age of 5 and in adolescence). The management of ischemic stroke in the paediatric age is often challenging because of pleomorphic age-dependent risk factors and aetiologies, high frequency of subtle or atypical clinical presentation, and lacking evidence-based data about acute recanalization therapies. Each pediatric tertiary centre should activate adequate institutional protocols for the optimization of diagnostic work-up and treatments.

Conclusion: The implementation of institutional standard operating procedures, summarizing the steps for the selection of candidate for neuroimaging among the ones presenting with acute neurological symptoms, may contribute to shorten the times for thrombolysis and/or endovascular treatments and to improve the long-term outcome.
\end{abstract}

\section{What is Known:}

- Acute ischemic stroke has a higher incidence in newborns than in older children (1/3500 live birth versus 1-2/100,000 per year).

-Randomized clinical trial assessing safety and efficacy of thrombolysis and/or endovascular treatment were never performed in children

What is New:

-Recent studies evidenced a low risk (2.1\% of the cases) of intracranial haemorrhages in children treated with thrombolysis.

- A faster access to neuroimaging and hyper-acute therapies was associated with the implementation of institutional protocols for the emergency management of pediatric stroke.

Keywords Stroke $\cdot$ Neuroimaging $\cdot$ Diagnostic protocol $\cdot$ Thrombolysis $\cdot$ Recanalization therapies $\cdot$ Children

$\begin{array}{ll}\text { Abbreviations } \\ \text { MRI } & \text { Magnetic resonance imaging } \\ \text { VIPS } & \text { Vascular Effect of Infection In Pediatric } \\ & \begin{array}{l}\text { Stroke Study } \\ \text { PHACE }\end{array} \\ & \begin{array}{l}\text { Posterior fossa brain malformations, Heman- } \\ \text { gioma, Arterial lesions, Cardiac abnormali- } \\ \text { ties, and Eye abnormalities }\end{array}\end{array}$

Communicated by Nicole Ritz

Vincenzo Leuzzi

vincenzo.leuzzi@uniroma1.it

Mario Mastrangelo

mario.mastrangelo@uniroma1.it

Laura Giordo

laura.giordo@uniroma1.it

Giacomina Ricciardi

giacomina.ricciardi@uniroma1.it
HSV1 Herpes simplex virus 1

HSV2 Herpes simplex virus 2

VZV Varicella-Zoster virus

TIA Transient ischemic attack

OR Odds ratio

CI Confidence interval

PedNIHSS Pediatric National Institute of Health Stroke Scale
Manuela De Michele

m.demichele@policlinicoumberto1.it

Danilo Toni

danilo.toni@uniroma1.it

1 Child Neurology and Infantile Psychiatry Unit, Department of Human Neuroscience, Sapienza University of Rome, Rome, Italy

2 Emergency Department Stroke Unit, Department of Human Neurosciences, Sapienza University of Rome, Rome, Italy 


$\begin{array}{ll}\text { ROSIER } & \begin{array}{l}\text { Recognition of Stroke in the Emergency } \\ \text { Room }\end{array} \\ \text { FAST } & \text { Face Arm Speech Test } \\ \text { CPSS } & \text { Cincinnati Prehospital Stroke Scale } \\ \text { CT } & \text { Computed tomography } \\ \text { DWI } & \text { Diffusion weighted imaging } \\ \text { ADC } & \text { Apparent diffusion coefficient } \\ \text { ASL } & \text { Arterial spin labelling } \\ \text { SCD } & \text { Sickle cell disease } \\ \text { ACA } & \text { Anterior cerebral artery } \\ \text { MCA } & \text { Middle cerebral artery } \\ \text { rTPA } & \text { Recombinant tissue plasminogen activator } \\ \text { TIPS } & \text { Thrombolysis in Pediatric Stroke } \\ \text { ECASS } & \text { European Cooperative Acute Stroke Study } \\ \text { ADAPT } & \text { A Direct Aspiration First Pass Technique } \\ \text { IQR } & \text { Interquartile range }\end{array}$

\section{Introduction}

According to the American Heart Association- American Stroke Association definition, ischemic stroke is an episode of neurological dysfunction caused by focal cerebral, spinal, or retinal infarction involving a specific vascular district, and presenting with symptoms lasting for more than $24 \mathrm{~h}$ or until death and neuroimaging, pathological, and/or other objective evidences of focal ischemic injuries [1].

The occurrence of ischemic stroke in pediatric age, despite its rarity, implicates age-dependent peculiarities in terms of risk-factors, etiopathogenesis, clinical presentations, and therapeutic approaches [2-10]. The lack of validated evidencebased data about thrombolytic and endovascular treatments in children represents the main limit towards the prevention of life-threatening or disabling sequelae [2].

The aim of this review is to provide an updated overview on the current evidences and an insight into the future perspective on the management of acute ischemic stroke in childhood. A detailed analysis of epidemiological, clinical presentation, and management of neonatal stroke was beyond the scope of the authors even if the readers may find useful updates in the provided references [7, 11-13]

\section{Epidemiology}

The interpretation of data on incidence and risk factors of acute ischemic stroke in children is complicated by several differences in methods (e.g., identification of the cases via diagnostic code searches, variable age-ranges of the studied cohorts, large predominance of retrospective studies on selected cohorts of patients) [2-8]. The reported incidence is higher in newborns than in older children (1/3500 live birth versus $1-2 / 100,000$ per year) with a ratio of approximately six times higher [2]. The peaks of incidence were evidenced in the perinatal period (5-13/100,000 live births), in children under the age of $5(0.38 / 100,000$ per year) and in adolescence (0.48-0.6/100,000 per year) [2-8]. Two recent prospective studies, in Canada and Germany, reported lower overall incidence ranges $(0.41 / 100,000-1.72 / 100,000$ children/year) even if the German study did not include newborns $[4,5]$.

A higher predisposition to an earlier onset was highlighted in Asian and black children, because of a higher incidence of concurrent chronic disorders (with a relative risk of 2.14 and 2.28, respectively, for Asian and black children, compared to 1.34 of other ethnicities) [8]. Data about the relationships between age of clinical presentation and sexes are discordant $[9,10]$. Two-old retrospective studies reported a slightly increased risk in males, even after stratification by age and aetiologies (relative risk of 1.25), while a more recent prospective population-based study performed in the South of England did not detect any statistically significant differences [8-10].

\section{Etiopathogenesis and risk factors}

Pediatric ischemic stroke may be caused by several factors inducing thrombo-embolic occlusions of cerebral blood vessels and the activation of a complex cascade of events resulting in a permanent brain damage [7, 8, 11-15].

The main risk factors in children older than 28 days and their respective frequencies are summarized in Fig. 1Attachment A. Non atherosclerotic arteriopathies, cardiac disorders, and prothrombotic states account for most of the cases, with a variable distribution of their frequency in different geographical areas or age-ranges [14].

Data collected from the International Pediatric Stroke Study, a worldwide prospective study on 676 pediatric patients between 0 and 18 years, evidenced a higher prevalence of acute systemic conditions (including dehydration, sepsis, fever, acidosis, shock, anoxia/asphyxia, viral gastroenteritis) in Asia and South America and a lower prevalence of arteriopathies in Asia and chronic systemic conditions (haematological, oncological, and genetic disorders) in Europe and Australia [15]. Non atherosclerotic arteriopathies were the predominant aetiology in all the age ranges, with the highest prevalence in children between 5 and 9 years old, while the highest prevalence of cardiac disorders and acute conditions were reported in patients under the age of $5[14,16]$. The term "non atherosclerotic arteriopathies" included a group of heterogeneous disorders resulting in lesions or structural abnormalities involving the cerebral blood vessels' wall as a consequence of infectious, parainfectious or inflammatory mechanisms but also genetic predisposition or vascular malformation (e.g., focal cerebral arteriopathy, PHACE, sickle cell disease, post-varicella arteriopathy, fibromuscular dysplasia) [17]. The VIPS (Vascular Effect of Infection In Pediatric 
Stroke Study) study identified viral infections in the prior week, recent vaccination, black ethnicity, and rural residence as risk factors for a higher occurrence of arterial ischemic stroke in children $[18,19]$. Serological evidence of recent, and mostly asymptomatic, herpesvirus infections were detected in $45 \%$ of the enrolled patients with a predominance of HSV1 and HSV 2 over VZV (respectively $24.5 \%$ versus $11.3 \%$ of the cases) [20].

A large prospective cohort study by DeVeber et al. that recruited 894 children with stroke in Germany, Canada, and UK, aiming to determine the association between prothrombotic conditions and risk of recurrent episodes of stroke, evidenced a recurrence rate of $17.9 \%$ between 1 day and 136 months after the first stroke [21]. The following conditions were identified as independent risk factors for recurrence: antithrombin deficiency (hazard ratio 3.9; 95\% confidence interval 1.4-10.9), increased Lipoprotein(a) (hazard ratio 2.3; 95\% confidence interval 1.3-4.1) and more than one prothrombotic marker (hazard ratio 1.9; 95\% confidence interval 1.1-3.2) [21].

Cardiac conditions associated with stroke encompass a wide spectrum of either acquired or congenital diseases. A study by Rodan et al., that recruited 135 patients with congenital heart diseases and a diagnosis of acute ischemic stroke from Canadian Pediatric Ischemic Stroke Registry, reported a 10 -year-recurrence rate of 27\% [22]. The most common associated risk factors included mechanical heart valve (hazard ratio $=8.8$ ), systemic infection (hazard ratio $=5.7$ ), and prothrombotic-state (hazard ratio $=2.9$ ) [22]. Asaki et al. identified two additional risk factors in a retrospective case-control study on 52 cardiopathic children who developed an arterial ischemic stroke after invasive procedures: length of ICU hospitalization and post-procedural infections [23].

\section{Clinical presentation}

Childhood stroke may present, similarly to adults, with several localizing signs and symptom (hemiparesis or hemifacial weakness, speech or language dysfunctions, vision disturbances, or ataxia) even if non localizing manifestations and seizures have a higher frequency in pediatric age, especially in children under the age of 6 . (Table 1) $[2,8,16]$. Focal signs (numbness or weakness) are the most common clinical presentations at the onset in all the age ranges [5, 8]. Diffuse signs have a more variable distribution in the different age ranges: seizures appear in more than half of infants under the age of 12 months (56-75\%), whereas headache is a frequent complaint in school-age children $(33-50 \%)[5,8]$. In the first months of life, seizures (mainly brief focal episodes) and tone abnormalities (either increased or decreased muscle tone) are frequent clinical hallmarks, while focal symptoms and signs of encephalopathy may occur in a lower proportion of the cases and silent episodes without relevant symptoms are also possible $[7,11,24]$.
The most used clinical adult stroke assessment tools were not extensively validated in pediatric patients, and their predictive value remains uncertain [13]. Moreover, these tools are insensitive of posterior circulation defects as they do not assess ataxia, dysarthria, and visual field disturbances [13]. Pediatric National Institute of Health Stroke Scale (PedNIHSS) is the standard tool for the emergency evaluation of stroke severity [3]. It is a score-based system exploring 11 neurological domains (level of consciousness, best gaze, visual function, signs of facial palsy, motor function of arms and legs, limb ataxia, sensory functions, language, dysarthria, and extinction/inattention) that was associated with an excellent interrater reliability in a multicenter prospective cohort performed by trained child neurologists in 2011[13]. Mackay and colleagues evaluated the applicability of ROSIER (Recognition of Stroke in the Emergency Room) and FAST (Face Arm Speech Test) scales in a cohort of radiologically confirmed children and found a sensitivity for stroke detection of $81 \%$ and $76 \%$, respectively [25]. The same group reported poor predictive values for ROSIER and Cincinnati Prehospital Stroke Scale (CPSS) in discriminating between stroke and mimics in children presenting with acute neurological symptoms [26].

In a prospective observational study of 287 children presenting to the emergency department with acute onset focal neurologic symptom, Mackay et al. observed that stroke accounted for $7 \%$ of the aetiologies (versus $28 \%$ for migraine, $15 \%$ for seizures, $10 \%$ for Bell palsy, and $6 \%$ of conversion disorders) [27]. In a subsequent study, the same group compared 102 children with ischemic stroke/TIA and 280 children with other neurological symptoms mimicking acute stroke. The clinical predictors that were significantly associated with stroke included normal clinical status in the week before presentation (OR 5.76, 95\% CI 2.25-14.79), arm and face weakness (OR 8.66, 95\% CI 2.50-30.02 and OR $2.94,95 \%$ CI 1.19-7.28, respectively), and inability to walk (OR 3.38, 95\% CI 1.54-7.42) [28]. Seizures and loss of consciousness were not independently associated either with stroke or with mimics, suggesting that these symptoms might not be used as discriminator for diagnosis [28].

\section{Neuroimaging}

The acquisition of urgent MRI images is always necessary to achieve a rapid differential diagnosis (in children strokemimics have a higher frequency than in adults) and to decide the more suitable acute therapy (after having identified the areas of ischemia, penumbra, and/or large vessel occlusion and after having excluded intracranial haemorrhages) (Fig. 1) [2]. Current guidelines state that brain MRI should always be preferred to CT because this last one has a poor sensitivity with a missed diagnosis in $44-83 \%$ of cases [2, 27, 29, 30]. Figure 2 shows some illustrative MRIs that were performed in our Institution. 


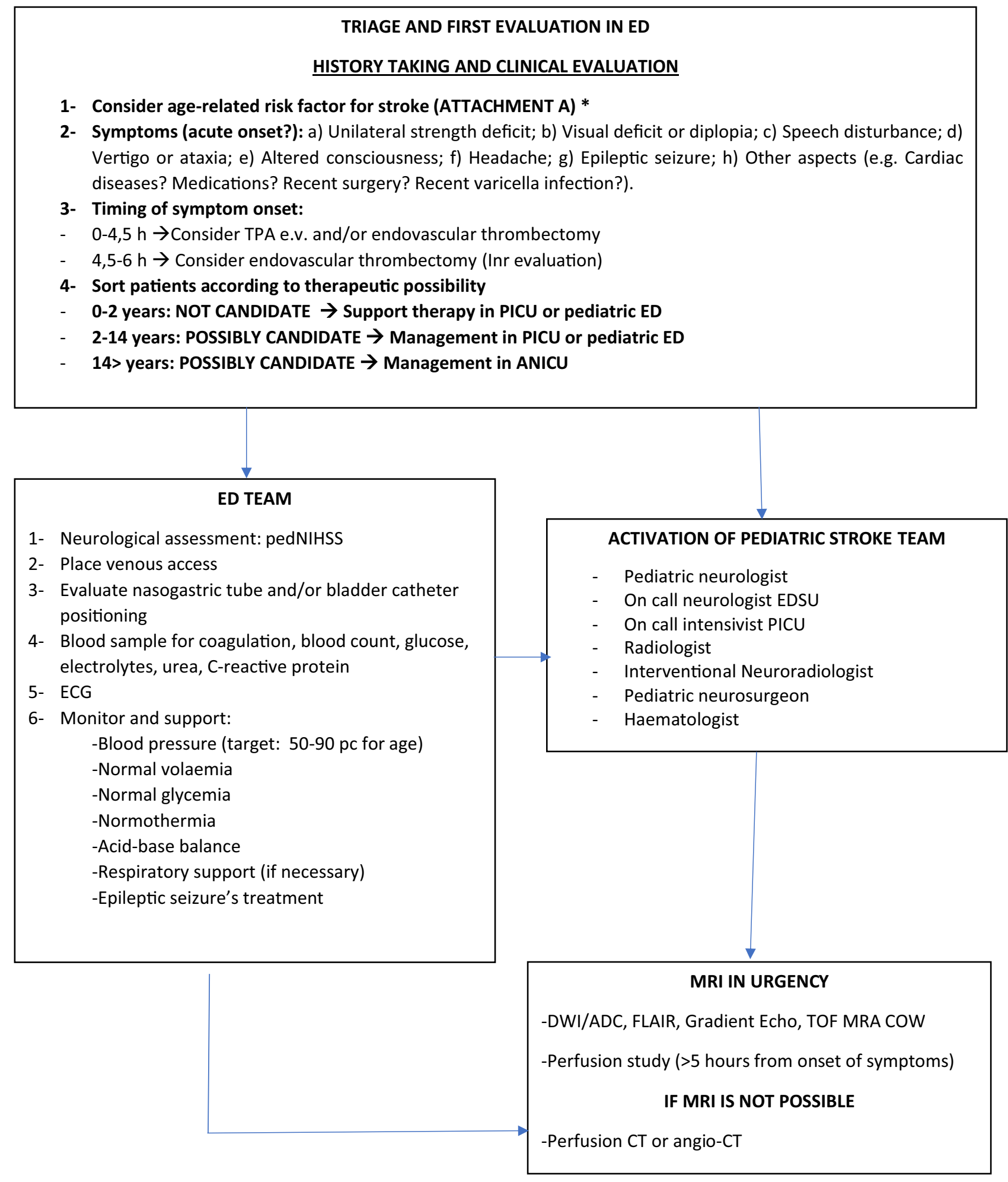

Fig. 1 A suggested protocol for the diagnostic work-up and the treatment of acute ischemic stroke in children. Legend: ED Emergency Department, rTPA recombinant tissue plasminogen activator, PICU pediatric intensive care unit, ANICU adults neurological intensive care unit, NICU neonatal intensive care unit, pedNIHSS Pediatric National Institute of Health Stroke Scale, ECG electrocardiogram, PCR protein $\mathrm{C}$ reactive, Inr interventional neuroradiologist, MRI magnetic resonance imaging, DWI diffusion weighted imaging,
ADC apparent diffusion coefficient, FLAIR fluid attenuated inversion recovery, TOF time of flight angiography, MRA magnetic resonance angiography, COW circle of Willis, CT computed tomography, ASA acetylsalicylic acid, PT prothrombin time, INR international normalized ratio, aPTT activated partial thromboplastine time, LP lumbar puncture, CNS central nervous system, MCA middle cerebral artery, LMWH low molecular-weight heparin 
Neuroimaging CRITERIA:

1- Excluded intracranial haemorrhage

2- Signs of ischemia

3- Large vessel occlusion (anterior and posterior circulation)

YES

INCLUSION CRITERIA FOR i.v. rTPA

1-within 4.5 hours of known onset of symptoms

2-more than two years of age

3-acute focal neurological deficit and pedNIHSS $>=6$ e

$$
<=24
$$

4- no contraindications (ATTACHMENT B)**

INCLUSION CRITERIA FOR ENDOVASCULAR THROMBECTOMY (ATTACHMENT C)***
NO

Hospitalization in NICU (age < 28 days) or PICU (ED if no bed available) or pediatric ward (if not critical conditions)

1- Neuroprotective or support therapy

2- Consider other invasive diagnostic procedures (angiography? Lumbar puncture?)

3- Endovascular therapy

4- Non-invasive diagnostic test to individuate etiology

\section{NO (at least one criterion not met)}

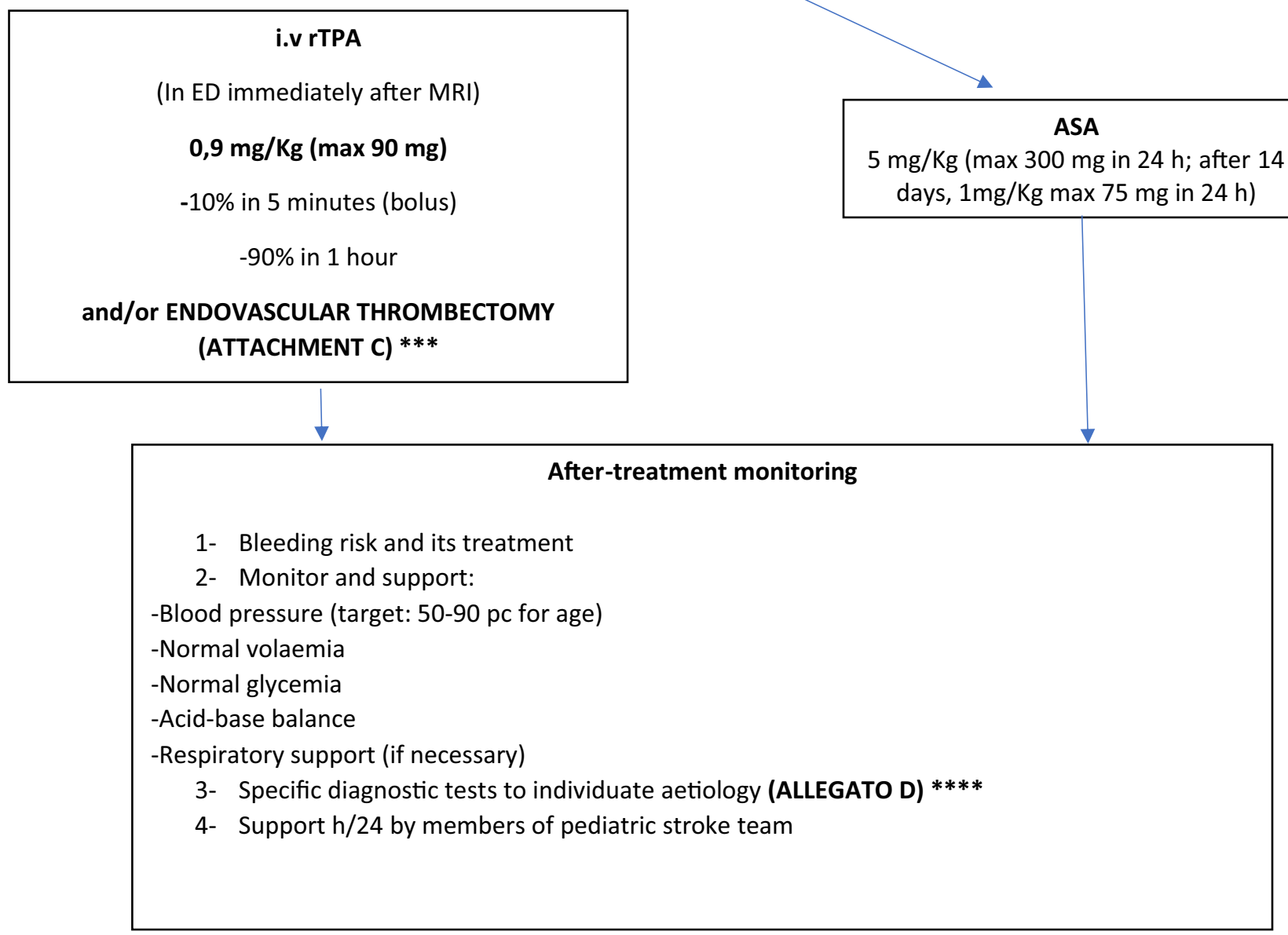

Fig. 1 (continued) 
ATTACHMENT A*: RISK FACTORS FOR CHILDHOOD ARTERIAL STROKE

0-28 days

See reference 2

28 days- 18 years

\begin{tabular}{|c|c|}
\hline \multirow{7}{*}{$\begin{array}{l}\text { Arteriopathies (intra and extracranial) } \\
21-53 \%\end{array}$} & Moya Moya \\
\hline & Sickle cell arteriopathy \\
\hline & Post- varicella arteriopathy \\
\hline & Arterial dissection \\
\hline & Primary CNS vasculitis \\
\hline & Para-/post infectious vasculitis \\
\hline & Fibromuscular dysplasia \\
\hline \multirow{6}{*}{$\begin{array}{l}\text { Cardiac } \\
24-31 \%\end{array}$} & Congenital/ acquired heart diseases \\
\hline & Patent foramen ovale (PFO) \\
\hline & Arrythmia \\
\hline & Previous cardiac surgery/ catheterization \\
\hline & Endocarditis \\
\hline & Other \\
\hline \multirow{9}{*}{$\begin{array}{l}\text { Hypercoagulable state } \\
28-13 \%\end{array}$} & Protein C deficiency \\
\hline & Protein S deficiency \\
\hline & Factor V Leiden mutation (G1691A) \\
\hline & Prothrombin mutation (PTG20210A) \\
\hline & MTHFR (C677T polymorphism) \\
\hline & Antithrombin III deficiency \\
\hline & Increased factor VIII \\
\hline & Hyperlipoproteinemia (alpha) \\
\hline & Lupus anticoagulant \\
\hline \multirow{5}{*}{$\begin{array}{l}\text { Acute systemic conditions } \\
22-9 \%\end{array}$} & Infectious diseases \\
\hline & Sepsis \\
\hline & Shock \\
\hline & Acidosis/Anoxia \\
\hline & Other \\
\hline \multirow{5}{*}{$\begin{array}{l}\text { Haemato oncological } \\
9-19 \%\end{array}$} & Sickle cell disease \\
\hline & Haemolytic/ iron deficiency anemia \\
\hline & Haematological malignancy \\
\hline & Solid extracranial tumors \\
\hline & Other \\
\hline \multirow{5}{*}{$\begin{array}{l}\text { Chronic head and neck disorders } \\
9-10 \%\end{array}$} & Brain tumors \\
\hline & Aneurysm/ arterio-venous malformations \\
\hline & Other cranial/ neck tumors \\
\hline & Migraine \\
\hline & Other \\
\hline \multirow{7}{*}{$\begin{array}{l}\text { Genetic/ metabolic } \\
1 \%\end{array}$} & Mitochondriopathy (e.g. MELAS, POLG1 mutation) \\
\hline & Fabry disease \\
\hline & PHACE syndrome \\
\hline & ACTA 2/ COL4A1 mutation \\
\hline & Trisomy 21 \\
\hline & Deficiency of Adenosine Deaminase 2 \\
\hline & $\begin{array}{l}\text { Connective tissue disorders (e.g. Ehlers Danlos- } \\
\text { /Marfan-/Loeys Dietz syndrome) }\end{array}$ \\
\hline \multirow{2}{*}{$\begin{array}{l}\text { Other } \\
10 \%\end{array}$} & Previous brain surgery \\
\hline & Trauma \\
\hline
\end{tabular}

Fig. 1 (continued) 


\section{ATTACHMENT B**: CONTRAINDICATIONS TO THROMBOLYSIS}

-Unknown time of symptoms onset

-Pregnancy (adolescents)

-Clinical presentation suggestive of subarachnoid haemorrhage (SAH), even if brain imaging is negative for blood

-Patient who would decline blood transfusion if indicated

-History of prior intracranial haemorrhage

-Known cerebral arterial venous malformation, aneurysm, or neoplasm

-Persistent systolic blood pressure more than $15 \%$ above the 95 th percentile for age while sitting or supine -Glucose less than $2.78 \mathrm{mmol} / \mathrm{L}$ or more than $22.22 \mathrm{mmol} / \mathrm{L}$

-Bleeding diathesis including platelets less than 100 000, prothrombin time (PT) more than15s (international normalised ratio (INR) more than 1.4), or elevated activated partial thromboplastin time (aPTT) more than upper limits of the normal range

-Clinical presentation consistent with acute myocardial infarction (MI) or post-MI pericarditis that requires evaluation by cardiology before treatment

-Prior stroke, major head trauma, or intracranial surgery within the past three months

-Major surgery within 10 days

-Parenchymal biopsy within 10 days (relative contraindication)

-Gastrointestinal or urinary bleeding within 21 days (relative contraindication)

-Arterial puncture at non-compressible site or LP within seven days (relative contraindication). Patients who have had a cardiac catheterization via a compressible artery are not excluded

-Patient with malignancy or within one month of completion of treatment for cancer

-Patients with an underlying significant bleeding disorder. Patients with a mild platelet dysfunction, mild von Willebrand disease, or other mild bleeding disorders are not excluded

Stroke related exclusion criteria:

-Mild deficit (Paediatric National Institute of Health Stroke Scale (PedNIHSS) less than 4) at start of tPA infusion or at time of sedation for neuroimaging, if applicable

-Severe deficit suggesting large territory stroke, with pre-tPA PedNIHSS more than 24, regardless of the infarct volume seen on neuroimaging

-Stroke suspected to be due to subacute bacterial endocarditis, Moya Moya, sickle cell disease, meningitis, bone marrow, air, or fat embolism

-Previously diagnosed primary angiitis of the central nervous system (PACNS) or secondary central nervous system (CNS) vasculitis. Focal cerebral arteriopathy of childhood is not a contraindication

\section{Neuroimaging related exclusion criteria:}

-Intracranial haemorrhage on pre-treatment head CT and MRI

-Intracranial dissection (defined as at or distal to the ophthalmic artery)

-Large infarct volume, defined by the finding of acute infarct on MRI involving one-third or more of the complete middle cerebral artery (MCA) territory involvement

\section{Drug-related exclusion criteria:}

-Known allergy to recombinant tissue plasminogen activator

-Patient who received heparin within four hours must have activated partial thromboplastin time (aPTT) in normal range

-Low molecular-weight heparin (LMWH) within past 24 hours (aPTT and INR will not reflect LMWH effect)

Fig. 1 (continued) 


\section{ATTACHMENT $C^{* * *}$}

A) INCLUSION CRITERIA FOR THROMBECTOMY

1. Persistent neurological deficit (PedNIHSS $\geq 6$ )

2. Onset of symptoms $<6$ hours

3. Radiologically confirmed large vessel occlusion (anterior or posterior circulation)

4. Age $\geq 2$ years

\section{B) AFTER-THROMBECTOMY MANAGEMENT}

1. Clinical monitoring of femoral artery access

2. Forced supine decubitus for at least 8 hours

3. Blood count control after 6 hours

4. Brain MRI after24 hours

5. Removal of the compression bondage after 12 hours

6. Inr available for emergencies

\section{ATTACHMENT D****: DIAGNOSTIC TESTS TO INDIVIDUATE ETIOLOGY (AFTER ACUTE PHASE OR AFTER TREATMENT)}

Blood second-level tests:

- $\quad$ Antibodies: aCL, $\beta 2 \mathrm{GPI}, \mathrm{LAC}, \mathrm{ANA}, \mathrm{dsDNA}$, ANCA, ENA

- $\quad$ C3, C4

- Total cholesterol, triglycerides, ApoA1 e B, Lipoprotein A

- Homocysteine and other specific metabolic tests (MTHFR, folate, B12, lactate, sialo transferrin isoelectrofocusing, enzymatic test for Fabry disease)

- Pathological haemoglobin

- Serology for varicella

- Ammonium

- $\quad$ Screening for Thrombophilia

- Pregnancy test

- $\quad$ Anti Xa (if monitoring EBPM)

- Urine tox screen

Medical consultations:

- Cardiological (ECG, transthoracic echocardiogram and, if necessary, transoesophageal echocardiogram)

- Infectious disease specialist (consider LP if meningoencephalitis is suspected)

Others (if indicated):

- $\quad$ EEG

- VEP

Fig. 1 (continued) 
Table 1 Frequency of signs and symptoms in children with arterial ischemic stroke $2,8,16$

Frequency of signs and symptoms in children with arterial ischemic stroke $(\%)$
Focal signs/symptoms $82-85 \%$
Seizures $15-31 \%$
Hemiparesis $72 \%$

Facial weakness $41 \%$

Speech disturbances $20-50 \%$

Visual disturbances 5-15\%

Ataxia 8-10\%

Other $19 \%$

Altered mental status $17-42 \%$

Vomiting $10 \%$

Papilledema $1 \%$

Other $8 \%$

Focal 20\%

Generalized $11 \%$

Both focal and generalized $2 \%$
Headache $23-50 \%$

"Hyperacute MRI protocols," including diffusion weighted imaging (DWI) and apparent diffusion coefficient maps (ADC) sequences, were developed also for children and demonstrated a good potential in detecting a reduced diffusivity in the involved arterial territories [31]. Perfusion MRI techniques, such as arterial spin labelling (ASL) sequences, can be appealing for children because allowing to measure relative cerebral blood flow and volume and detect areas of ischemia without the use of a contrast agent [32]. ASL has been demonstrated to correlate with the degree of stenosis, infarct location, diffusion restriction, and follow-up T2 infarct volumes [32].

The distribution of the lesions might help physicians to identify several common aetiologies occurring in the pediatric age [33]. For instance, strokes in children with cardiac disorders are often bilateral, involving both anterior and posterior circulation, with an increased tendency to a hemorrhagic transformation [33]. Herpesviruses-related strokes are generally multifocal, with an involvement of the limbic system and basal ganglia associated with leptomeningeal enhancement, reflecting meningitis [33]. The sickle cell disease (SCD)-related strokes are generally located at the border-zone between the ACA and MCA territories, with relative sparing of the posterior circulation [34].

\section{Treatment}

Randomized clinical trial assessing safety and efficacy of thrombolysis and/or endovascular treatment were never performed for pediatric age $[35,36]$.

The most updated pediatric stroke guidelines state that it is feasible to apply rTPA in children from 2 years of age, with persistent disabling neurological deficits (e.g., Pediatric
NIH Stroke Scale score $\geq 6$ at the time of intervention) and radiographically confirmed cerebral large artery occlusion, within $4.5 \mathrm{~h}$ of known onset of symptoms [2, 35, 36]. The suggested drug dosage is the same used for adults, although according to known age-related differences in the fibrinolytic system with children having higher levels of tissue plasminogen inhibitor than adults, it would be reasonable to consider that children might benefit of higher dosages of rTPA [2, 13].

The Thrombolysis in Pediatric Stroke (TIPS) trial was designed as a 5-year prospective multicentre trial to study the safety and pharmacokinetic profile of intravenous rTPA in pediatric patients with acute ischemic stroke [37]. Although it was closed prematurely due to lack of enrolment, it favoured the formation of paediatric stroke centres and acute paediatric stroke protocols [37]. A 2020 study by Amlie-Lefond et al. retrospectively collected data on children treated with intravenous rTPA for arterial ischemic stroke in 16 former TIPS sites [38]. It showed that the overall risk of symptomatic intracerebral haemorrhage after intravenous rTPA, when given within $4.5 \mathrm{~h}$ after symptom onset, was as low as $2.1 \%$ (compared with $6.4 \%$ reported for adults) [38, 39]. Symptomatic intracerebral haemorrhages were defined if ECASS (European Cooperative Acute Stroke Study) II parenchymal hematoma type 2 or any intracranial haemorrhage associated with neurological deterioration within 36 were observed after tPA administration [39].

Several differences in adult versus childhood stroke might influence the performance of endovascular thrombectomy (i.e., large vessel occlusion, collateral vessel status, intracranial vessel sizes in children) [2, 40]. The 2020 Save ChildS study provided multicentre evidence for the use of mechanical thrombectomy in children with large vessel occlusion ischemic stroke [39]. This study was focused on the potential effect modification of different thrombectomy techniques, (A Direct Aspiration First Pass Technique [ADAPT] and nonADAPT groups) as well as different device sizes, suggesting that the global neurological outcome was generally positive regardless of any specific device and approach selection [41]. No remarkable differences were found between ADAPT and non-ADAPT group in terms of rate of successful recanalization $(85.7 \%$ versus $87.5 \%$ ), PedNIHSS on day 7 , and modified Rankin scale at the discharge and after 6 and 24 months [41].

No new insights were developed in the last decades about standard of cares when thrombolysis, or endovascular treatments are not feasible with most of the suggested protocols being based on traditional anti-thrombotic therapies (mainly low-molecular weight heparins and acetylsalicylic acid) [2, $35,36]$. The lacking initiation of an anti-thrombotic treatment for stroke prevention is associated with a 1.5-2.5 fold increased risk of recurrences after a first episode [2]. Antithrombotic therapies are contraindicated in the acute setting when haemorrhagic stroke has not been excluded and in other 
Fig. 2 A Right parietal FLAIR-hyperintense lesion in a 14-year-old girl who presented with a complete anaesthesia of the left forearm and hand, visual impairment and headache. B Left temporo-occipital T2-lesion in a 6-year-old boy who presented with aphasia. $\mathbf{C}$ and $\mathbf{D}$ Angio-MRI and PWI sequences in a 2-year-old boy with the occlusion of M1 tract of the left-middle cerebral artery and of the A1 tract of the ipsilateral anterior cerebral artery
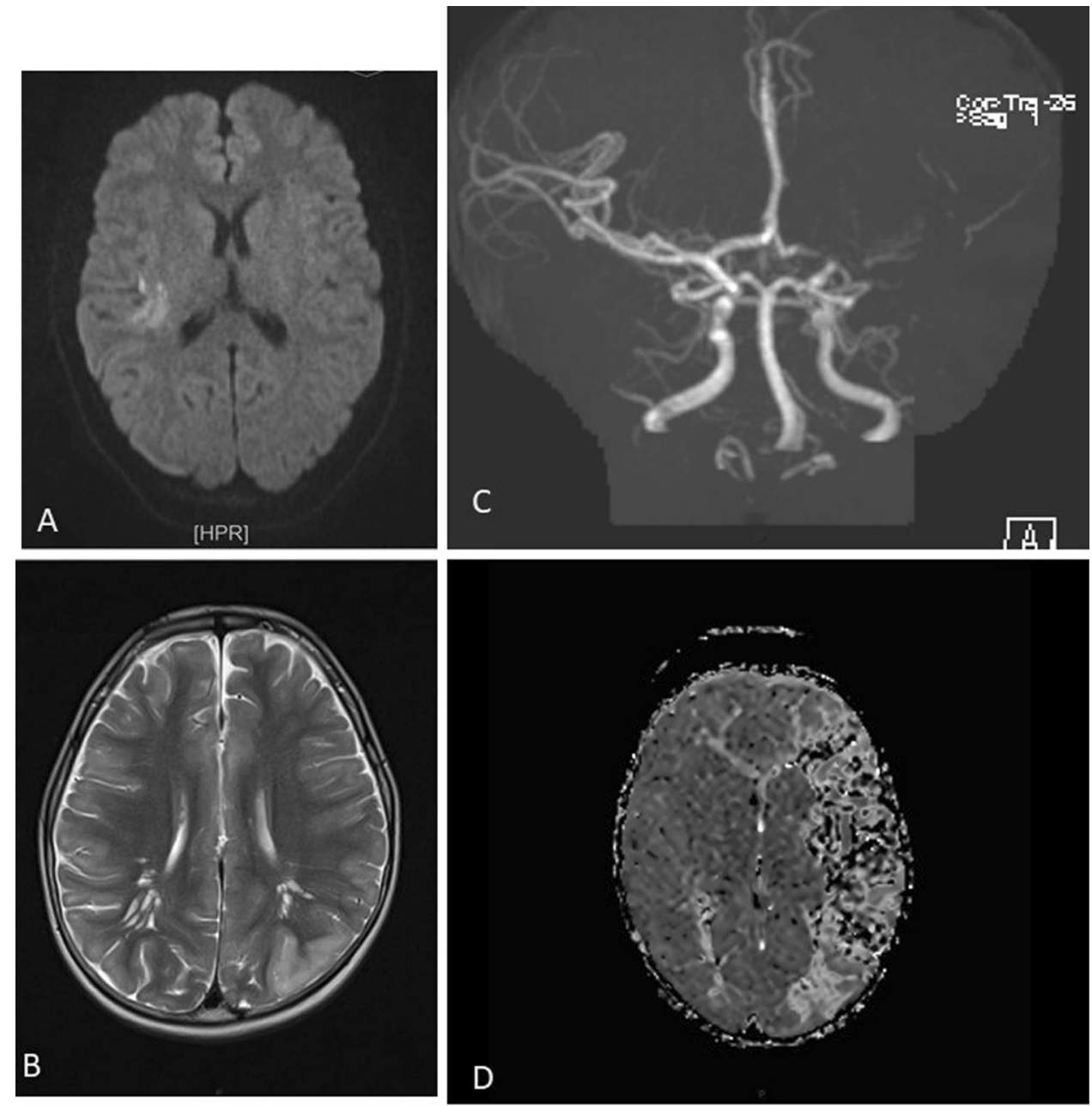

cases including Moya Moya disease, surgery within the previous $24 \mathrm{~h}$, methotrexate toxicity, thrombocytopenia with platelet count less than $50,000 / \mathrm{mm}^{3}$, or history of heparininduced thrombocytopenia. [2].

The long-term treatment is commonly based on lowmolecular weight heparins or acetylsalicylic acid without clear data suggesting which one of the two therapies is better in children and safety data supporting both approaches [2]. A longer-term prevention therapy should be considered in patients with genetically determined thrombophilia (e.g., hyperhomocysteinemia, protein $\mathrm{C}$ deficiency, antithrombin deficiency) or in children with a stroke following congenital heart diseases, while in the other cases, the duration of the treatment may depend on the underlying aetiology with a usual preferred temporal range between 2 years and a lifetime [2]. No data about the prophylactic use of direct oral anticoagulants (e.g., antithrombin agent or anti-factor Xa agents) in the paediatric age were collected [2].

Specific prevention therapies may be considered for selected aetiologies (e.g., transfusions or hydroxyurea for sickle cell disease, L-arginine for MELAS, agalsidase or migalastat for Fabry disease, pyridoxine in combination with folic acid and vitamin B12, methionine-restricted, cystinesupplemented diet and betaine, for homocystinuria) even if adequate supporting evidences are often not available because of their rare occurrence [2, 42]. The usefulness of other specific strategies (e.g., anticoagulation for pediatric arterial dissection, patent foramen ovale closure, steroids for focal cerebral arteriopathies, or surgical strategies for moyamoya) remain unsolved controversial [2].

\section{Management protocols}

A suggested protocol for the early evaluation, treatment, and diagnostic work-up of children with ischemic arterial stroke, that was developed in our institution, is illustrated in Fig. 1.

The activation and implementation of paediatric acute stroke protocols speed up stroke recognition and diagnosis in children, making the delivery of hyperacute recanalization treatment feasible [43-49]. A recent paper by Rivkin et al. retrospectively compared children presenting with acute neurological symptoms to their tertiary children's hospital 


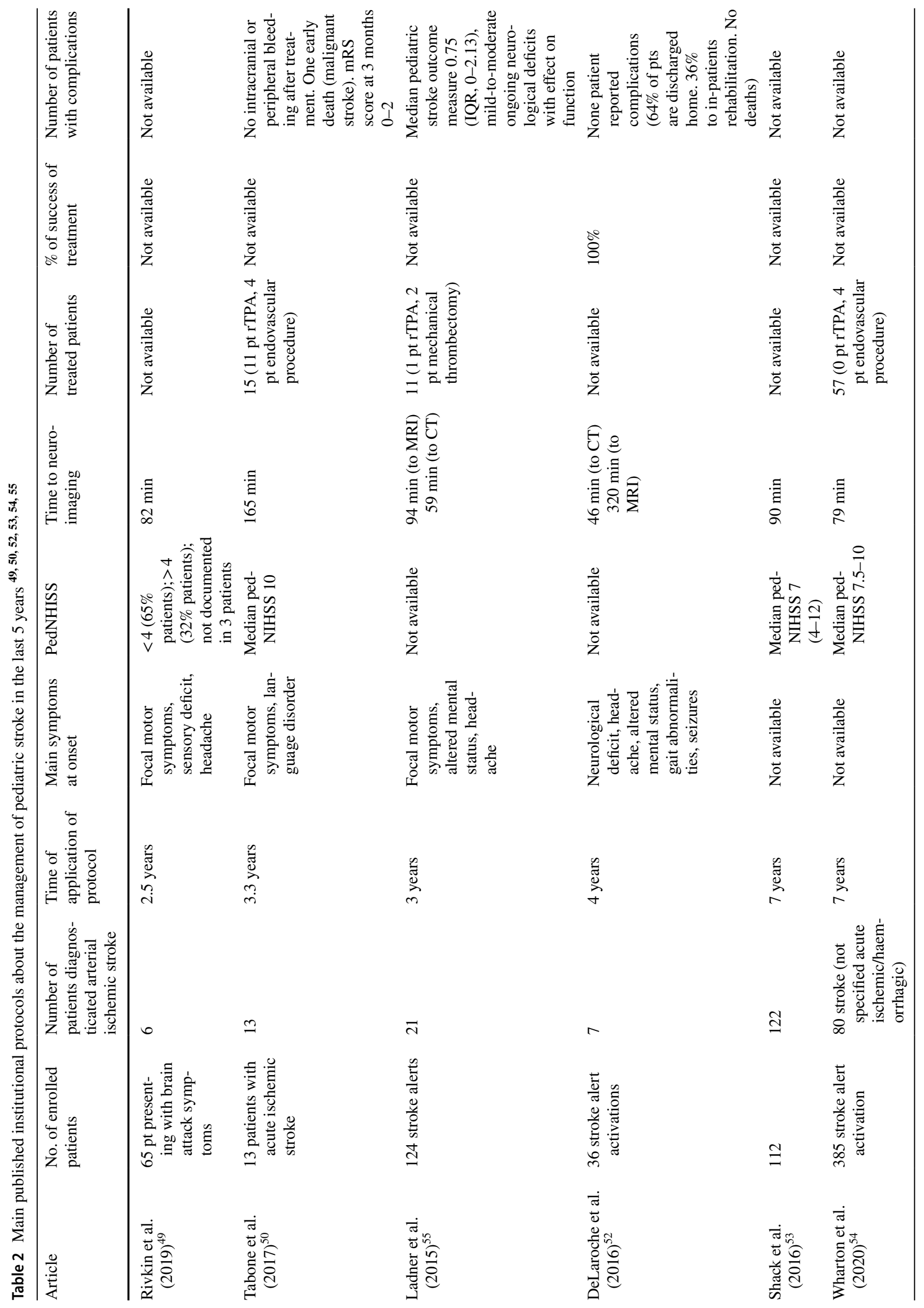


before and after the implementation of their stroke alert system [45]. The median time from emergency department arrival to neuroimaging was significantly lower for patients evaluated after the implementation of the stroke pathway (82 min; IQR, 54-123 min after [ $n=65]$ vs $196 \mathrm{~min}$; IQR, $85-230 \mathrm{mi}$ before $[n=14] ; P<0.01$ ), thus enabling a greater proportion of them to lay within the time frame for acute intervention [43]. Similar results have been published by Tabone et al. with a reported mean time of $165 \mathrm{~min}$ from symptoms onset to MRI for 13 consecutive children who underwent acute recanalization treatments (i.v. rTPA, endovascular procedure or both) as part of a regional pediatric acute stroke protocol in France [44].

The most recent protocols have all been similarly developed from multidisciplinary working groups involving paediatric and adult specialists (emergency physicians, stroke unit fellows, neurologists, interventional and diagnostic neuroradiologists, anaesthesiologists) [43-46]. All the protocols were organized in three diagnostic steps based on (a) the quick assessment of the patient at the point of care (either emergency departments, intensive care units, or in-hospital wards), (b) the transfer to the MRI suite where pre-determined hyperacute MRI stroke protocols are being performed, and (c) the determination of eligibility for recanalization therapy, according to the presence/absence of inclusion/exclusion criteria (see Attachment $\mathrm{C}$ in Fig. 1) [51-54]. Table 2 summarizes the protocols that were published in the last 5 years [43, 44, 46-49]. The application of all these protocols resulted in a neuroradiologically confirmed diagnosis in almost all the patients within the correct time window for the application of acute recanalization therapies [43, 44, 46-49]. Despite these data, relevant differences in the treatment of choice emerged among the involved institutions, where the expertise for thrombolysis/thrombectomy was not always available [43-49]. None of the studies provided adequate information about the proportion of successfully treated patients, the frequency of treatment-related complications, and long-term outcome [43-49].

\section{Outcome}

Recent studies reported a mortality rate after childhood stroke between 2.6 and 5\% [4, 50]. The large Canadian prospective stroke registry documented the emergence of persistent neurological deficits in $60 \%$ of neonates and $70 \%$ of children, of which, $36 \%$ were mild, $23 \%$ were moderate, and $10 \%$ were severe deficits [4]. In older children, the observed deficits remained mostly stable (with a reduction in their deficit severity in $16 \%$ and an increase in $8 \%$ of them) and recurrent arterial ischemic stroke or TIA were reported in $12 \%$ of the cases [4]. In another large population-based cohort study, Fullerton et al. observed that although recurrences were rare after perinatal stroke, one-fifth of arterial ischemic stroke recurred in later childhood (with a 5-year cumulative recurrence rates of $1.2 \%$ after perinatal stroke and $19 \%$ after later childhood stroke) [51].

A recent review of 22 studies on newborns and children with stroke evidenced lower executive functions performances than typically developing patients [52]. The longterm evolution of executive functions impairment was scarcely explored (only two longitudinal studies with small cohorts of patients), and no concordant evidence were collected about a possible impact of factors such as age at stroke onset, time since stroke, and lesion characteristics [52]. Inhibition resulted as more impaired than working memory processes or cognitive flexibility, independently by the age at stroke onset or lesion features [52].

\section{Conclusions}

Physicians approaching to ischemic pediatric stroke should consider several differences versus adults that represent remarkable clues for the diagnosis and the treatment: (1) the more significant etiopathogenic role of intracranial non-atherosclerotic arteriopathies, thromboembolic complications of congenital cardiopathies, and haematological disorders such as sickle cell's disease or coagulopathies; (2) the high frequency of atypical presentations in children (e.g., headache only or no evident clinical symptoms); and (3) the lack of validated guidelines for thrombolytic and endovascular treatments in paediatric age with obvious negative consequences on the prevention of permanent neurological sequelae.

Authors' contributions -Mario Mastrangelo planned the realization of the review, wrote the first draft, and revised the paper. Laura Giordo, Giacomina Ricciardi, and Manuela De Michele realized the literature searches and the data analysis and revised the manuscript. Danilo Toni and Vincenzo Leuzzi revised the manuscript as senior authors.

Funding Open access funding provided by Università degli Studi di Roma La Sapienza within the CRUI-CARE Agreement.

\section{Declarations}

Conflict of interest The authors declare no competing interests.

Open Access This article is licensed under a Creative Commons Attribution 4.0 International License, which permits use, sharing, adaptation, distribution and reproduction in any medium or format, as long as you give appropriate credit to the original author(s) and the source, provide a link to the Creative Commons licence, and indicate if changes were made. The images or other third party material in this article are included in the article's Creative Commons licence, unless indicated otherwise in a credit line to the material. If material is not included in the article's Creative Commons licence and your intended use is not permitted by statutory regulation or exceeds the permitted use, you will 
need to obtain permission directly from the copyright holder. To view a copy of this licence, visit http://creativecommons.org/licenses/by/4.0/.

\section{References}

1. Sacco RL, Kasner SE, Broderick JP et al (2013) An updated definition of stroke for the 21 st century: a statement for healthcare professionals from the American Heart Association/American Stroke Association. Stroke 44:2064-2089

2. Ferriero HJ, Fullerton et al (2019) Management of stroke in neonates and children a scientific statement from the American Heart Association/American Stroke Association. on behalf of the American Heart Association Stroke Council and Council on Cardiovascular and Stroke Nursing. Stroke 50:e00-e00

3. Dunbar M, Kirton A (2018) Perinatal stroke: mechanisms, management, and outcomes of early cerebrovascular brain injury. Lancet Child Adolesc Health 2(9):666-676

4. deVeber GA, Kirton A, Booth FA et al (2017) Epidemiology and outcomes of arterial ischemic stroke in children: the Canadian Pediatric Ischemic Stroke Registry. Pediatr Neurol 69:58-70

5. Gerstl L, Weinberger R, Heinen F et al (2019) Arterial ischemic stroke in infants, children, and adolescents: results of a Germanywide surveillance study 2015-2017. J Neurol 266(12):2929-2941

6. Rambaud T, Legris N, Bejot B et al (2020) Acute ischemic stroke in adolescents. Neurology 94:e1-e12

7. Grunt S, Mazenauer L, Buerki SE et al (2015) Incidence and outcomes of symptomatic neonatal arterial ischemic stroke. Pediatrics 135(5):e1220-e1228

8. Mallick A, Ganesan V, Kirkham FJ et al (2014) Childhood arterial ischaemic stroke incidence, presenting features, and risk factors: a prospective population-based study. Lancet Neurol 13:35-43

9. Fullerton HJ, Wu YW, Zhao S, Johnston SC (2003) Risk of stroke in children: ethnic and gender disparities. Neurology 61:189-194

10. Golomb MR, Fullerton HJ, Nowak-Gottl U, Deveber G (2009) Male predominance in childhood ischemic stroke: findings from the International Pediatric Stroke Study. Stroke 40:52-57

11. Martinez-Biarge M, Ferriero DM, Cowan FM (2019) Perinatal arterial ischemic stroke. Handbook of clinical neurology, Vol. 162 (3rd series) Neonatal Neurology

12. Harteman JC, Groenendaal F, Kwee A et al (2012) Risk factors for perinatal arterial ischaemic stroke in full-term infants: a casecontrol study 97(6):F411-F416

13. Royal College of Pediatrcs and Child Health and the Stroke Association Stroke in childhood (2017) Clinical guidelines for diagnosis, management and rehabilitation. NICE accredited. Royal college of pediatrics and child health. Royal College of Pediatrcs and Child Health Ed. London

14. Gerstl L, Weinberger R, von Kries R et al (2018) Risk factors in childhood arterial ischaemic stroke: findings from a populationbased study in Germany. Eur J Paediatr Neurol 22(3):380-386

15. Mackay MT, Wiznitzer M, Benedict SL et al (2011) Arterial ischemic stroke risk factors: the International Pediatric Stroke Study. Ann Neurol 69(1):130-140

16. Felling RJ, Rafay MF, Bernard TJ et al (2020) Predicting recovery and outcome after pediatric stroke: results from the international pediatric stroke study. Ann Neurol 87(6):840-852

17. Numis AL, Fox CK (2014) Arterial ischemic stroke in children: risk factors and etiologies. Curr Neurol Neurosci Rep 14(1):422

18. Mackay MT, Steinlin M (2019) Recent developments and new frontiers in childhood arterial ischemic stroke. Int J Stroke 14(1):32-43

19. Fullerton HJ, Hills NK, Elkind MS et al (2015) Infection, vaccination, and childhood arterial ischemic stroke: results of the VIPS study. Neurology 85:1459-1466
20. Elkind MS, Hills NK, Glaser CA et al (2016) Herpesvirus infections and childhood arterial ischemic stroke: results of the VIPS study. Circulation 133(8):732-741

21. DeVeber G, Kirkham F, Shannon K et al (2019) Recurrent stroke: the role of thrombophilia in a large international pediatric stroke population. Haematologica 104(8):1676-1681

22. Rodan L, McCrindle BW, Manlhiot C et al (2012) Stroke recurrence in children with congenital heart disease. Ann Neurol 72(1):103-111

23. Asakai H, Stojanovski B, Galati JC, Zannino D, Cardamone M, Hutchinson D, Cheung MMH, Mackay MT (2017) Risk factors for peri-procedural arterial ischaemic stroke in children with cardiac disease. Pediatr Cardiol 38:1385-1392

24. Kirton A, Armstrong-Wells J, Chang T et al (2011) Symptomatic neonatal arterial ischemic stroke: the international pediatric stroke study. Pediatrics 128:e1402-e1410

25. Yock-Corrales A, Babl FE, Mosley IT et al (2011) Can the FAST and ROSIER adult stroke recognition tools be applied to confirmed childhood arterial ischemic stroke? BMC Pediatr 21(11):93

26. Mackay MT, Churilov L, Donnan GA, Babl FE, Monagle P (2016) Performance of bedside stroke recognition tools in discriminating childhood stroke from mimics. Neurology 86(23):2154-2161

27. Mackay MT, Chua ZK, Lee M et al (2014) Stroke and nonstroke brain attacks in children. Neurology 82:1434-1440

28. Mackay MT, Yock-Corrales A, Churilov L et al (2016) Differentiating childhood stroke from mimics in the emergency Department. Stroke 47:2476-2481

29. Rafay MF, Pontigon AM, Chiang J et al (2009) Delay to diagnosis in acute pediatric arterial ischemic stroke. Stroke 40(1):58-64

30. Srinivasan J, Miller SP, Phan TG, Mackay MT (2009) Delayed recognition of initial stroke in children: need for increased awareness. Pediatrics 124(2): e227-e234

31. Mirsky DM, Beslow LA, Amlie-Lefond C et al (2017) Pathways for neuroimaging of childhood stroke. Pediatr Neurol 69:11-23

32. Chen J, Licht DJ, Smith SE et al (2009) Arterial spin labeling perfusion MRI in pediatric arterial ischemic stroke: initial experiences. J Magn Reson Imaging 29:282-290

33. Zuccoli G, Fitz C, Greene S, Cummings DD et al (2018) Imaging review of common and rare causes of stroke in children. Top Magn Reson Imaging 27(6):463-477

34. Zhang X, Li C, Li Q (2016) Magnetic resonance imaging in pediatric sickle cell anemia. Exp Ther Med 12(2):555-558

35. Pacheco JT, Siepmann T, Barlinn J et al (2018) Safety and efficacy of recanalization therapy in pediatric stroke: a systematic review and meta-analysis. Eur J Paediatr Neurol 22(6):1035-1041

36. Bhatia K, Kortman H, Blair C et al (2019) Mechanical thrombectomy in pediatric stroke: systematic review, individual patient data meta-analysis, and case series. J Neurosurg Pediatr. 1-14

37. Chan AK, Hovinga CA, Gill JC et al (2015) Thrombolysis in pediatric stroke study. Stroke 46(3):880-885

38. Amlie-Lefond C, Shaw DWW, Cooper A et al (2020) Risk of intracranial hemorrhage following intravenous tPA (tissue-type plasminogen activator) for acute stroke is low in children. Stroke 51(2):542-548

39. The National Institute of Neurological Disorders and Stroke rt-PA stroke study group. (1995). Tissue plasminogen activator for acute ischemic stroke. N Eng1 J Med. 333:1581-1587.

40. Barry M, Hallam DK, Bernard TJ, Amlie-Lefond C (2019) What is the role of mechanical thrombectomy in childhood stroke? Pediatr Neurol 95:19-25

41. Sporns PB, Straeter R, Minnerup J et al (2020) Does device selection impact recanalization rate and neurological outcome? An analysis of the Save ChildS Study. Stroke 51(4):1182-1189

42. Testai FD, Gorelick PB (2010) Inherited metabolic disorders and stroke part 1: Fabry disease and mitochondrial myopathy, encephalopathy, lactic acidosis, and strokelike episodes. Arch Neurol 67:19-24 
43. Harrar DB, Salussolia CL, Kapur K et al (2020) A stroke alert protocol decreases the time to diagnosis of brain attack symptoms in a pediatric emergency department. J Pediatr 216:136-141.e6

44. Tabone L, Mediamolle N, Bellesme C et al (2017) Regional pediatric acute stroke protocol: initial experience during 3 years and 13 recanalization treatments in children. Stroke 48:2278-2281

45. Rivkin MJ, Bernard TJ, Dowling MM, Amlie-Lefond C (2016) Guidelines for urgent management of stroke in children. Pediatr Neurol 56:8-17

46. DeLaroche AM, Sivaswamy L, Farooqi A, Kannikeswaran N (2016) Pediatric stroke clinical pathway improves the time to diagnosis in an emergency department. Pediatr Neurol 65:39-44

47. Shack M, Andrade A, Shah-Basak PP et al (2017) Acute Stroke Protocol study group. A pediatric institutional acute stroke protocol improves timely access to stroke treatment. Dev Med Child Neurol. 59:31-37

48. Wharton JD, Barry MM, Lee CA et al (2020) Pediatric acute stroke protocol implementation and utilization over 7 years. J Pediatr 220:214-220
49. Ladner TR, Mahdi J, Gindville MC et al (2015) Pediatric acute stroke protocol activation in a children's hospital emergency department. Stroke 46(8):2328-2331

50. Beslow LA, Dowling MM, Hassanein SMA et al (2018) International pediatric stroke study investigators. Mortality after pediatric arterial ischemic stroke. Pediatrics. 141: e20174146

51. Fullerton HJ, Wu YW, Sidney S, Johnston SC (2007) Risk of recurrent childhood arterial ischemic stroke in a population-based cohort: the importance of cerebrovascular imaging. Pediatrics 119(3):495-501

52. Rivella C, Viterbori P (2021) Executive function following pediatric stroke. A systematic review Child Neuropsychol 27:209-231

Publisher's Note Springer Nature remains neutral with regard to jurisdictional claims in published maps and institutional affiliations. 